\title{
AVALIAÇÃO DA EFICIÊNCIA DE ACARICIDAS, POR AÇÃO DIRETA E RESIDUAL, SOBRE Tetranychus mexicanus (McGREGOR, 1950) (ACARI: TETRANYCHIDAE) EM CITROS
}

\author{
Daniel Júnior de Andrade ${ }^{1}$, Carlos Amadeu Leite de Oliveira ${ }^{1}$, Fernando César Pattaro ${ }^{1}$
} 'Depto. de Fitossanidade - FCAV/UNESP - Via de acesso Prof. Paulo Donato Castellane, s/n - CEP: 14870-000,
Jaboticabal - SP. Endereço eletrônico do primeiro autor: danieldwv@yahoo.com.br

\section{RESUMO}

A utilização de agrotóxicos é praticamente a única tática eficiente de controle dos ácaros tetraniquídeos na cultura dos citros. O objetivo deste trabalho foi avaliar a eficiência de acaricidas sobre Tetranychus mexicanus, em diferentes dosagens, por ação direta e residual, para selecionar produtos eficientes para o controle do ácaro e utilizá-los adequadamente num programa de manejo da resistência. Os tratamentos estabelecidos foram constituídos dos acaricidas, expressos em $\mathrm{mL}$ ou g do produto por $100 \mathrm{~L}$ de água: cyhexatin a $50 \mathrm{~mL}$, óxido de fenbutatin a $80 \mathrm{~mL}$, hexythiazox a $3 \mathrm{~g}$, dinocap a $200 \mathrm{~mL}$, propargite a $100 \mathrm{~mL}$, pyridaben a $50 \mathrm{~mL}$ e abamectin a 50 $\mathrm{mL}$, em pré-mistura com $250 \mathrm{~mL}$ de óleo vegetal, enxofre a $400 \mathrm{~g}$, sulfurado inorgânico a 5.000 $\mathrm{mL}$ e testemunha. Com base nos resultados, concluiu-se que os produtos dinocap, propargite, pyridaben, abamectin + óleo vegetal e sulfurado inorgânico se mostraram os mais eficientes no controle do ácaro T. mexicanus. Enxofre e cyhexatin apresentaram média eficiência no controle do T. mexicanus. Óxido de fenbutatin e hexythiazox não foram eficientes no controle do ácaro $T$. mexicanus.

Palavras-chave: Controle químico, acaridas, manejo da resistência.

\section{EVALUATION OF THE EFFICIENCY OF MITICIDES, BY DIRECT AND RESIDUAL ACTION, ON Tetranychus mexicanus (McGREGOR, 1950) (ACARI: TETRANYCHIDAE) MITE ON CITRUS}

\begin{abstract}
The use of traditional pesticides is practically the only efficient tactics of control of the spider mite in the citrus crop. The objective of this work was to evaluate the efficiency of miticides on Tetranychus mexicanus, in different dosages for direct and residual action for assist in the strategies of handling of the resistance. The treatments have been composed for the following products, expressed in $\mathrm{mL}$ or $\mathrm{g}$ of the product in 1001 of water: cyhexatin in $50 \mathrm{~mL}$ oxide of fenbutatin in $80 \mathrm{~mL}$, hexythiazox in $3 \mathrm{~g}$, dinocap in $200 \mathrm{~mL}$, propargite in $100 \mathrm{~mL}$, pyridaben in $50 \mathrm{~mL}$, abamectin in $50 \mathrm{~mL}$ in pre-mixture with $250 \mathrm{~mL}$ of vegetable oil, sulfur in $400 \mathrm{~g}$, sulphurated inorganic in $5,000 \mathrm{ml}$ and control. Based on the results was concluded that the products dinocap, propargite, pyridaben, abamectin + vegetable oil and sulphurated inorganic were most efficient ones in the control of $T$. mexicanus. Sulfur and cyhexatin presented mean efficency in the control of $T$. mexicanus. Oxide of fenbutatin and hexythiazox did not present satisfactory efficiency to control $T$. mexicanus.
\end{abstract}


Key words: chemical control, miticides, handling of the resistance.

\section{INTRODUÇÃO}

O ácaro Tetranychus mexicanus (McGregor, 1950) (Acari: Tetranychidae) foi relatado primeiramente nos Estados Unidos da América e, posteriormente, no México e Argentina. No Brasil, foi referido por Flechtmann \& Paschoal (1967), em plantas cítricas, onde tece grandes quantidades de teias nas folhas, acarretando intensa desfolha e prejudicando o desenvolvimento das plantas, motivo pelo qual, se tem justificado a aplicação de acaricidas. A espécie tem, provavelmente, os citros como principais hospedeiros (Rodrigues \& Oliveira, 2005).

Em citros, sua incidência é mais comum em mudas, em condições de viveiro. As fêmeas são esverdeadas com manchas escuras no dorso, medem aproximadamente $0,50 \mathrm{~mm}$ e reproduzem-se sexuadamente ou por partenogênese arrenótoca, dando origem a machos. O ciclo é completado em aproximadamente 18 dias para o macho e 19,5 dias para a fêmea, a temperaturas de 19 a $25^{\circ} \mathrm{C}$ (Paschoal, 1968). Os ovos são esféricos e ligeiramente 'amarelados, comumente suspensos nas teias (Rodrigues \& Oliveira, 2005).

Nas regiões citrícolas do Estado de São Paulo, sua ocorrência é maior nos meses secos do ano, que correspondem ao período de inverno (Oliveira, 1993). Infesta principalmente a superfície abaxial das folhas e as depressões do fruto, causando ligeira descoloração do tecido afetado, que se intensifica com o aumento populacional (Flechtmann \& Paschoal, 1967).

$O$ uso de agrotóxicos tem sido praticamente a única tática de controle dos ácaros tetraniquídeos na cultura dos citros, embora não exista nenhum produto registrado nos órgãos competentes para recomendação de controle desses ácaros. No entanto, a evolução da resistência de ácaros fitófagos aos acaricidas em curto intervalo de tempo depende, dentre outros fatores, de seus usos freqüentes. Torna-se, então, de suma importância a adoção de estratégias de anti-resistência, como a rotação de acaricidas de grupos distintos, visando a diminuir a pressão de seleção (Stark et al., 1997; Stumpf et al., 2001).

O objetivo deste trabalho foi avaliar a eficiência de acaricidas sobre T. mexicanus, em diferentes dosagens, por ação direta e residual em condições de laboratório para serem utilizados em programas de controle, considerando-se o manejo da resistência.

\section{MATERIAL E MÉTODOS}

Os bioensaios foram realizados no Laboratório de Acarologia do Departamento de Fitossanidade da Faculdade de Ciências Agrárias e Veterinárias, Universidade Estadual Paulista, Câmpus de JaboticabalSP.

Inicialmente, foram coletadas folhas de citros altamente infestadas por $T$. mexicanus, em pomares localizados na Faculdade de Ciências Agrárias e Veterinárias, os quais não vinham sendo pulverizados com qualquer tipo de agrotóxico por vários meses. As folhas foram fixadas em mudas de citros com aproximadamente um ano de idade e mantidas em câmara climatizada a $25 \pm 1^{\circ} \mathrm{C}$, umidade relativa de $60 \pm 5 \%$ e fotofase de 14 horas, para início da criação-estoque.

O delineamento experimental utilizado nos bioensaios de ação direta e residual foi o inteiramente casualizado, com dez tratamentos repetidos quatro vezes, sendo cada repetição composta por um disco de folha de citros (Citrus sinensis da variedade Pêra) de $2,5 \mathrm{~cm}$ de diâmetro, totalizando 40 discos (Tabela 1). 
Tabela 1. Relação dos produtos utilizados nos bioensaios de ação direta e residual sobre adultos de T. mexicanus.

\begin{tabular}{cccc}
\hline Tratamentos & \multicolumn{2}{c}{ Dosagens } & \\
\cline { 1 - 3 } Ingrediente ativo & mL ou g & Kg ou L & Nome comercial \\
p.c./100L & p.c./2.000L & \\
\hline Cyhexatin & 50 & 1 & Sipcatin 500 SC \\
Óxido de fenbutatin & 80 & 1,6 & Torque \\
Hexythiazox & 3 & 0,06 & Savey PM \\
Dinocap & 200 & 4 & Karathane CE \\
Propargite & 100 & 2 & Omite720 CE \\
Pyridaben & 50 & 1 & Sanmite \\
Abamectin + óleo** & $50+250$ & $1+5$ & Kraft36 CE \\
Enxofre & 400 & 8 & Kumulus GD \\
Sulforado inorgânico & 5000 & 100 & Calda sulfocálcica \\
Testemunha & - & - & - \\
\hline Testemunha & - & &
\end{tabular}

* Testemunha sem aplicação de acaricida

** Óleo vegetal ( Natur'l óleo ).

Os discos de folha foram obtidos através de um vazador circular de metal e colocados com a superfície abaxial voltada para cima em placas de Petri de $9 \mathrm{~cm}$ de diâmetro por $2 \mathrm{~cm}$ de altura, sobre uma camada fina de algodão hidrófilo, que foi mantido umedecido.

Para avaliar a ação direta dos produtos, foram transferidos 10 ácaros adultos de $T$. mexicanus procedentes da criação-estoque para cada disco de folha, com auxílio de pincel de apenas um pêlo, e utilizando-se de microscópio estereoscópico.

Para a pulverização dos discos com os vários produtos, utilizou-se de um micropulverizador (Torre de Potter), calibrado a $10 \mathrm{lbf} / \mathrm{pol}^{2}$, gastando-se $2 \mathrm{~mL}$ de calda por aplicação, o qual proporcionava uma cobertura uniforme sobre a superfície tratada. Após as pulverizações, os discos foram mantidos em câmara climatizada às mesmas condições de temperatura, umidade relativa e luz citadas anteriormente.

A ação direta dos produtos sobre o acarino foi avaliada $24 ; 48$ e 72 horas após a aplicação, retiraram-se os ácaros mortos, vivos e retidos na camada de algodão. Após a última avaliação, foram transferidos outros 10 ácaros adultos para cada um dos discos para avaliar a ação residual, também após 24, 48 e 72 horas após a transferência dos ácaros.

Os dados relativos às contagens de ácaros foram transformados em $\ln (\mathrm{x}+5)$, com o objetivo de normalizar os dados a serem analisados pelo teste F. As médias foram comparadas pelo teste de Tukey, a 5\% de probabilidade. A redução real ou a eficiência foi calculada pela fórmula de Abbott (1925), tomando-se por base a população de ácaros vivos de cada tratamento e transformados em porcentagem de sobrevivência.

\section{RESULTADOS E DISCUSSÃO}

Vinte e quatro horas após a aplicação direta dos produtos sobre T. mexicanus, nos tratamentos com óxido de fenbutatin $\mathrm{e}$ hexythiazox, o número médio de ácaros 
vivos não diferiu estatisticamente $\mathrm{da}$ testemunha, situação que perdurou até 48 horas após a aplicação (Tabela 2). Contudo, 72 horas após a aplicação dos produtos sobre os ácaros, todos os tratamentos diferiram estatisticamente da testemunha em relação ao número médio de ácaros, com exceção do tratamento com hexythiazox (Tabela 2).

Segundo Gravena (1994), desde os anos 80 , o acaricida hexythiazox tem sido utilizado para o controle de ácaros em pomares de citros no Brasil. Esse produto apresenta ação sobre ovos e formas imaturas de diferentes espécies de ácaros (Campos \& Omoto, 2006), além de mostrar seletividade a inimigos naturais de pragas (Sato et al., 1992). Vale salientar que hexythiazox é utilizado em mistura com outros acaricidas de ação adulticida com relativo sucesso (Campos \& Omoto, 2006).

Tabela 2. Número médio de ácaros T. mexicanus vivos relativos às avaliações realizadas $24 ; 48 \mathrm{e}$ 72 horas após a aplicação no bioensaio de ação direta e após a transferência dos ácaros no bioensaio de ação residual.

\begin{tabular}{|c|c|c|c|c|c|c|c|c|c|}
\hline \multirow{3}{*}{ Tratamentos } & \multicolumn{5}{|c|}{ Acão direta } & \multicolumn{4}{|c|}{ Ação residual } \\
\hline & \multicolumn{5}{|c|}{ Horas após a aplicação } & \multicolumn{4}{|c|}{ Horas após a transferência } \\
\hline & 24 & 48 & & \multicolumn{2}{|l|}{72} & 24 & 48 & \multicolumn{2}{|l|}{72} \\
\hline Cyhexatin & $7,0 \mathrm{~B}$ & $5,5 \quad \mathrm{C}$ & $\mathrm{C}$ & 4,3 & $\mathrm{C}$ & $8,2 \mathrm{~A}$ & $5,8 \mathrm{AB}$ & \multicolumn{2}{|l|}{$4,0 \quad \mathrm{~B}$} \\
\hline Ó. de fenbutatin & $9,5^{\mathrm{A}}$ & $7,5 \mathrm{~B}$ & B & \multicolumn{2}{|c|}{$6,5 \mathrm{~B}$} & $10,0 \mathrm{~A}$ & $9,8 \mathrm{~A}$ & \multirow{2}{*}{\multicolumn{2}{|c|}{$\begin{array}{r}9,8 \mathrm{~A} \\
10,0 \mathrm{~A}\end{array}$}} \\
\hline Hexythiazox & $9,0 \mathrm{AB}$ & $9,0 \mathrm{AB}$ & & \multicolumn{2}{|l|}{$9,0 \mathrm{AB}$} & $10,0 \mathrm{~A}$ & $10,0 \mathrm{~A}$ & & \\
\hline Dinocap & $0,2 \quad \mathrm{D}$ & 0,0 & $\mathrm{E}$ & 0,0 & $\mathrm{D}$ & $0,5 \quad \mathrm{~B}$ & $0,0 \quad \mathrm{C}$ & 0,0 & $\mathrm{C}$ \\
\hline Propargite & $0,0 \quad \mathrm{D}$ & 0,0 & $\mathrm{E}$ & 0,0 & $\mathrm{D}$ & $0,0 \quad B$ & 0,0 & 0,0 & $\mathrm{C}$ \\
\hline Pyridaben & $0,0 \quad \mathrm{D}$ & 0,0 & $\mathrm{E}$ & 0,0 & $\mathrm{D}$ & $0,8 \quad \mathrm{~B}$ & 0,0 & 0,0 & $\mathrm{C}$ \\
\hline Abamectin+óleo & 0,0 & 0,0 & $\mathrm{E}$ & 0,0 & $\mathrm{D}$ & $2,0 \quad \mathrm{~B}$ & 1,0 & 0,8 & $\mathrm{BC}$ \\
\hline Enxofre & $3,8 \quad \mathrm{C}$ & 3,5 & $\mathrm{D}$ & 2,8 & $\mathrm{C}$ & $3,5 \quad \mathrm{~B}$ & $2,3 \quad \mathrm{BC}$ & 2,0 & $\mathrm{BC}$ \\
\hline S. inorgânico & $0,0 \quad \mathrm{D}$ & 0,0 & $\mathrm{E}$ & 0,0 & $\mathrm{D}$ & $8,0 \mathrm{~A}$ & $5,2 \mathrm{AB}$ & 0,8 & $\mathrm{BC}$ \\
\hline Testemunha & $10,0^{\mathrm{A}}$ & $10,0 \mathrm{~A}$ & & $9,5 \mathrm{~A}$ & & $9,75 \mathrm{~A}$ & $9,2 \mathrm{~A}$ & $9,0 \mathrm{~A}$ & \\
\hline TESTE F & $212,6^{* *}$ & 245,8 & & $113,7^{* *}$ & & $22,0 * *$ & $28,5^{* *}$ & $23,8 * *$ & \\
\hline C.V. \% & 3,3 & 3 , & & 4,2 & & 9,0 & 8,3 & 5 & \\
\hline
\end{tabular}

Medias seguidas por letras iguais na mesma coluna não diferem entre si, pelo teste de Tukey, a $5 \%$ de probabilidade. Para a comparação das médias, os dados originais foram transformados em $\ln (\mathrm{X}+5)$.

** Significativo pelo teste $\mathrm{F}$, a $5 \%$ de probabilidade.

Os resultados obtidos 24 horas após a transferência dos ácaros T. mexicanus para a verificação da ação residual dos acaricidas, mostram que os tratamentos cyhexatin, óxido de fenbutatin, hexythiazox e sulfurado inorgânico não diferiram estatisticamente da testemunha em relação ao número médio de ácaros vivos (Tabela 2). Cyhexatin e óxido de fenbutatin são acaricidas organoestânicos que, segundo Corbett et al. (1984), agem por meio da inibição da fosforilação oxidativa, ou seja, de modo distinto dos demais produtos utilizados em citros. Assim, a rotação de acaricidas organoestânicos com outros grupos de acaricidas torna-se altamente recomendável (Konno et al., 2001).

$\mathrm{Na}$ tabela 3 são apresentadas as porcentagens de mortalidade ou de eficiência dos produtos por ação direta e residual sobre T. mexicanus. 
Tabela 3. Porcentagem de mortalidade dos produtos no controle de T. mexicanus por ação direta e residual.

\begin{tabular}{ccccccc}
\hline & \multicolumn{3}{c}{ Ação direta } & \multicolumn{3}{c}{ Ação residual } \\
\cline { 2 - 7 } Tratamentos & \multicolumn{3}{c}{ Horas após a aplicação } & \multicolumn{3}{c}{$\begin{array}{c}\text { Horas após a } \\
\text { transferência }\end{array}$} \\
\cline { 2 - 7 } & $\mathbf{2 4}$ & $\mathbf{4 8}$ & $\mathbf{7 2}$ & $\mathbf{2 4}$ & $\mathbf{4 8}$ & $\mathbf{7 2}$ \\
\hline Cyhexatin & 30,0 & 45,0 & 55,3 & 15,9 & 37,8 & 55,8 \\
Óxido de fenbutatin & 5,0 & 25,0 & 31,6 & 0,0 & 0,0 & 0,0 \\
Hexythiazox & 10,0 & 10,0 & 5,3 & 0,0 & 0,0 & 0,0 \\
Dinocap & 97,5 & 100,0 & 100,0 & 94,9 & 100,0 & 100,0 \\
Propargite & 100,0 & 100,0 & 100,0 & 100,0 & 100,0 & 100,0 \\
Pyridaben & 100,0 & 100,0 & 100,0 & 92,3 & 100,0 & 100,0 \\
Abamectin+óleo & 100,0 & 100,0 & 100,0 & 79,0 & 89,2 & 91,7 \\
Enxofre & 62,5 & 65,0 & 71,1 & 64,1 & 74,9 & 77,8 \\
Sulfurado inorgânico & 100,0 & 100,0 & 100,0 & 17,9 & 43,2 & 91,7 \\
Testemunha & - & - & - & - & - & - \\
\hline
\end{tabular}

A redução real foi calculada pela fórmula de Abbott $\mathrm{RR}=[(\%$ sobrev. test. - \%sobrev. trat.) $/ \%$ sobrev. test. $] \times 100$ sobrev. = sobrevivência; test. $=$ testemunha; trat.= tratamento.

Após 24 horas da aplicação, no bioensaio de ação direta, os produtos dinocap, propargite, pyridaben, abamectin + óleo e sulfurado inorgânico acarretaram mortalidade superior a $97,5 \%$, evidenciando excelente ação de choque desses produtos sobre adultos de T. mexicanus. O tratamento com enxofre mostrou-se com eficiência intermediária por ação de choque em relação aos demais acaricidas com percentuais de mortalidade de $62,5 \%$. No entanto, os tratamentos com cyhexatin e óxido de fenbutatin apresentaram baixa eficiência, com percentuais de mortalidade de 30 e $5 \%$, respectivamente (Tabela 3 ).

$\mathrm{Na}$ avaliação realizada 48 horas após a aplicação, observou-se um ligeiro aumento da eficiência do cyhexatin, óxido de fenbutatin e enxofre; enquanto, para hexythiazox, a eficiência manteve-se em 10\%. Decorridas 72 horas após a aplicação sobre os ácaros, verificou-se aumento da porcentagem de mortalidade nos tratamentos com cyhexatin, óxido de fenbutatin e enxofre; em contrapartida, houve redução da mortalidade no tratamento hexythiazox. Esse fato pode ser explicado devido à redução da população no tratamento-testemunha, proporcionalmente maior que a redução da população no tratamento com hexythiazox, pois a fórmula de Abbott (1925) leva em consideração a sobrevivência de ácaros do tratamento com acaricida e testemunha no momento da avaliação.

Por ação residual, constatou-se, 24 horas após a transferência, altas porcentagens de redução nos tratamentos com propargite, dinocap e pyridaben, cujos percentuais variaram de 92,3 a $100 \%$. Abamectin + óleo e enxofre reduziram a população do ácaro em 79 e 64,1\%, respectivamente. Os demais apresentaram baixa ação residual após as 24 horas da exposição.

A eficiência dos produtos manteve-se semelhante após 48 horas.

Decorridas 72 horas após a transferência dos ácaros para os discos de folha, os tratamentos com dinocap, propargite, pyridaben, abamectin + óleo e sulfurado inorgânico apresentaram mortalidades superiores a $91,7 \%$, concordando com os resultados obtidos no bioensaio de ação direta.

Os dados obtidos para o sulfurado inorgânico corroboram a pesquisa de 
Andrade et al. (2007), que verificaram alta eficiência residual do sulfurado inorgânico sobre $T$. mexicanus (acima de $75 \%$ ), que é maior à medida que aumenta o período de exposição do acarino sobre o resíduo do produto. Oliveira et al. (2002) também verificaram que o sulfurado inorgânico apresenta elevada eficiência residual sobre o ácaro da falsa ferrugem Phyllocoptruta oleivora. Porém, Pattaro (2003) observou que o sulfurado inorgânico, em dosagens de até $4.000 \mathrm{~mL} / 100 \mathrm{~L}$ de água, apresenta baixa ação residual sobre adultos de $B$. phoenicis.

Cyhexatin e enxofre mostraram intermediária mortalidade residual, após 72 horas da transferência, com percentuais de 55,8 e $77,8 \%$, respectivamente. Verificou-se também que os tratamentos com óxido de fenbutatin e hexytriazox não apresentaram ação residual sobre ácaros adultos.

Ressalta-se que já foi relatada a resistência cruzada entre os acaricidas organoestânicos cyhexatin, óxido de fenbutatin e azocyclotin em Tetranychus urticae Koch, na Austrália, e entre cyhexatin e óxido de fenbutatin em $T$. urticae e Tetranychus pacificus McGregor, nos Estados Unidos. A evolução da resistência de T. urticae a propargite, em macieira, foi observada na Austrália e, em rosa, na Nova Zelândia, enquanto a evolução de resistência de Panonychus ulmi a este produto, também em macieira foi observada na Nova Zelândia (Franco, 2002; Franco et al., 2007).

Estudos de resistência dos acaricidas que se mostraram eficientes para o controle do $T$. mexicanus, neste trabalho devem ser realizados visando à implementação de um sistema adequado de manejo.

\section{CONCLUSÕES}

- Dinocap, propargite, pyridaben, abamectin + óleo vegetal e sulfurado inorgânico mostraram-se os mais eficientes no controle do ácaro $T$. mexicanus em citros, tanto por ação direta quanto por ação residual.

- Dinocap, propargite, pyridaben, abamectin + óleo vegetal e sulfurado inorgânico poderiam ser utilizados num programa de controle do ácaro mexicano $T$. mexicanus em citros, embora estes produtos ainda não estejam registrados para essa finalidade.

- Enxofre e cyhexatin apresentaram média eficiência no controle do $T$. mexicanus, todavia respeitando os princípios de manejo de resistência.

- Óxido de fenbutatin e hexythiazox não foram eficientes no controle do ácaro $T$. mexicanus.

\section{REFERÊNCIA BIBLIOGRÁFICA}

ABBOTT, W. S. A method of computing the effectiveness of an insecticide. Journal of Economic Entomology, Lanham, v. 18, p. $265-267,1925$.

ANDRADE, D.J.; OLIVEIRA, C.A.L.de; ROMANI, G.N. PATTARO, F.C. Efeito da calda sulfocálcica sobre o ácaro Tetranychus mexicanus (McGregor, 1950) em citros. Revista de Agricultura, Piracicaba, v.82, p.161-169, 2007.

CAMPOS, F.J.; OMOTO, C. Estabilidade da resistência de Brevipalpus phoenicis (Geijskes) (Acari: Tenuipalpidae) a hexythiazox em pomares de citros. Neotropical Entomology, Londrina, v. 35, p. 840-848, 2006.

CORBETT, J. R.; WRIGHT, K.; BAILLIE, A.C. The biochemical mode of action of pesticides. London: Academic Press, 1984. 382p.

FLECHTMANN, C.H.W.; PASCHOAL, A.D. Os ácaros dos citros. $O$ solo, Piracicaba, v.2, p.53-56,1967.

FRANCO, C.R. Detecção e caracterização da resistência de Brevipalpus phoenicis (Geijskes, 1939) (Acari: Tenuipalpidae) 
ao acaricida propargite. 2002. 64p. Dissertação de Mestrado, Escola Superior de Agricultura "Luiz de Queiroz", USP, Piracicaba.

FRANCO C.R.; CASARIN, N. F. B.; DOMINGUES, F. A.; OMOTO, C. Resistência de Brevipalpus phoenicis (Geijskes) (Acari: Tenuipalpidae) a acaricidas inibidores da respiração celular em citros: Resistência cruzada e custo adaptativo. Neotropical Entomology, Londrina, v. 36, p. 565-576, 2007.

GRAVENA, S. Manejo integrado de pragas em citros no Brasil: uma visão atual. In: L.C. DONADIO \& S. GRAVENA (coords.), Manejo integrado de pragas dos citros. SEMINÁRIO INTERNACIONAL DE CITROS - MIP. 3. Campinas, Fundação Cargill, 1994. p.41-56. (Anais).

KONNO, R.H., C.R. FRANCO \& C. OMOTO. Suscetibilidade de populações de Brevipalpus phoenicis (Geijskes, 1939) (Acari: Tenuipalpidae) a acaricidas organoestânicos em citros. Scientia Agrícola, Piracicaba, v.58, p.703-709, 2001.

OLIVEIRA, C.A.L. de. Ácaros dos citros. BASF, 1993. 18p. (Boletim Técnico).

OLIVEIRA, M.L.; PATTARO, F.C.; OLIVEIRA, C.A.L. de. Influência do resíduo de calda sulfocálcica na eficiência do Vertimec $18 \mathrm{CE}$ no controle de Phyllocoptruta oleivora, em citros. In: CONGRESSO BRASILEIRO DE ENTOMOLOGIA, 19. 2002, Manaus, p.252. (Resumos).

PASCHOAL, A.D. Sobre a biologia do ácaro Tetranychus mexicanus
(Acarina:Tetranychidae). $\quad$ O Solo, Piracicaba, v.1, p.67-70, 1968.

PATTARO, F.C. Calda sulfocálcica no agrossistema citrícola. 2003. 73p. Dissertação (Mestrado em Agronomia) Faculdade de Ciências Agrárias e Veterinárias, Universidade Estadual Paulista, Jaboticabal-SP.

STARK, J.D.; TANIGOSHI, L.; BOUNFOUR, M.; ANTONELLI, A. Reproductive potential: its influence on the susceptibility of a species to pesticides. Ecotoxicology and Environmental Safety, USA, v.37, p.273-279, 1997.

STUMPF, N.; NAUEN, R. Cross resistance, inheritance, and biochemistry of mitocondrial electron transport inhibitoracaricide resistance in Tetranychus urticae (Acari: Tetranychidae). Journal of Economic Entomology, Lanham, v.94, p.1.577$1.583,2001$.

RODRIGUES, J.C.V.; OLIVEIRA, C.A.L. de. Ácaros Fitófagos dos citros. p. 689727. In: MATTOS JUNIOR, D. de; NEGRI, J. D. de; PIO, R. M.; POMPEU JUNIOR, J. (coords.). Citros. Campinas: Instituto Agronômico e Fundag. 2005. 929p.

SATO, M.E.; RAGA, A.; CERÁVOLO, L.; ROSSI, A.C.; CEZÁRIO, A.C. Efeito de acaricidas sobre Brevipalpus phoenicis (Geijskes, 1939) (Acari: Tenuipalpidae) e ácaros predadores (Família Phytoseiidae) em citros. Revista Brasileira de Fruticultura, Cruz das Almas, v.14, p.97-93, 1992. 